\title{
Interactive comment on "Scale dependence of cirrus heterogeneity effects. Part I: MODIS thermal infrared channels" by Thomas Fauchez et al.
}

\section{Anonymous Referee \#1}

Received and published: 11 March 2017

\section{General comments}

The paper compares 3D and 1D Monte Carlo simulations of a cirrus cloud field at four different MODIS wavelength channels in the thermal spectral range. The aim of the study is to investigate the difference in brightness temperature between 1D and 3D radiative transfer in an inhomogeneous cloud field from a nadir satellite perspective and to find the optimal horizontal resolution where the error between the realistic $3 \mathrm{D}$ radiative transfer and the commonly used $1 \mathrm{D}$ approximations are at a minimum. Simulations of different horizontal resolutions $(50 \mathrm{~m}$ to $10 \mathrm{~km})$ have been performed and differences due to horizontal transport of radiation and the averaging/aggregation of high resolution pixels to coarser resolution, the plane parallel bias, have been addressed. 
It was shown that the optimal horizontal resolution varies between 100 and $250 \mathrm{~m}$, depending on the wavelength channel. Even at this optimal resolution the difference in brightness temperatures between the $1 \mathrm{D}$ and $3 \mathrm{D}$ radiative transfer simulation can be up to $7 \mathrm{~K}$.

Additionally, sensitivity tests for varying optical properties have been performed.

The off nadir perspective was addressed by simulating one of the four MODIS channels of this study.

With this study, the authors extend former work in this field by showing the difference between 1D and 3D RT brightness temperatures at different horizontal resolutions. The paper is suitable for publication after minor revision.

\section{General Comments:}

The optical thickness used in the paper is not always defined. In one figure, the optical thickness at $0.86 \mu \mathrm{m}$ is shown, while most of the manuscript refers to the $12.03 \mu \mathrm{m}$ optical thickness. It is not always mentioned which optical thickness is used for the comparisons. The authors might clarify which optical thickness is used where in the study. I would recommend using a single one. What is the reason for choosing that specific wavelength optical thickness?

The authors refer often to the mean path of a photon/FLIP when effects of the horizontal resolution are concerned. It might help readers to have a certain number associated with the mean path at the four different wavelengths considered in the work. Maybe the value of the mean path at a certain optical thickness (e.g. 1 or 1.4 as this seems to be the mean optical thickness of the cirrus cloud in this study) could be added. 
Some of the figures are hard to read. Especially the choice of red and pink in many of ACPD the line plots make it difficult to see the difference in the results. Please see the more specific comments below.

Many abbreviations are introduced in the introduction. Sometimes the authors use capital letters to show the origin of the abbreviation, but not throughout the text. I recommend doing this throughout the text.

How much different would results of a simulation of the $8.52 \mu \mathrm{m}$ channel in the off nadir perspective be? As this channel has a stronger scattering, one might expect stronger 3D effects? I understand that these simulations are expensive, but it might be worth adding this channel to the analysis, or discuss possible differences in the results.

The 'Conclusion' in its current form is a summary of the shown work. An outlook and some discussion about the implications of the results is wanting. Please see the more specific comment below.

\section{Specific comments}

1) Page 2, Line 1: Delete "due"

2) Page 2, Line 6: Change "of their optical properties" to "cirrus cloud optical properties"

3) Page 2, Line 15-18: This part is challenging to read and understand. I guess that the authors want to point out that the thermal infrared spectral range should (next to the retrieval of temperature/pressure and altitude) also be used for the retrieval of 
optical properties such as COD and CED? This is part of the motivation for the study and should be pointed out more clearly.

4) Page 2, Line 19: Comma is missing: "AVHRR,"

5) Page 2, Line 20: delete brackets: ((Garnier et al., 2012, 2013))

Interactive

comment

6) Page 2, Line 21: example concerning the capital letters mentioned above: "Optimal Estimation Method" (OEM)

7) Page 2, Line 28: "etc.": The authors might add additional reasons or change the sentence to: "due to time constraints on 3-D forward radiative calculations and the lack of..."

8) Page 3, Line 1: Is longwave here the same as thermal IR?

9) Page 3, Line 1: Is the cooling rate in 1D too high or too low by $10 \%$ ?

10) Page 3, Line 13: delete PPHB; it is already introduced at this point.

11) Page 5, Line 27: optical thickness at which wavelength?

12) Page 6, Line 10: Delete sentence "Note that TIR retrieval techniques are often limited to effective diameters between 5 and $50 \mu \mathrm{m}$." either here or in line 5/6 above. 
13) Page 6, Line 30: "cirrus 1" - There is only one cirrus case used in this study. I recommend deleting "cirrus 1 " in the whole manuscript. Otherwise one would expect more than one scene.

14) Page 7, Line 18/19: The authors might mention the FLIP mean path as a second motivation for the $50 \mathrm{~m}$ resolutions already at this point. I saw that it is mentioned later in the text, but it would already be worthy here.

15) Page 7, Line 21: Mention the wavelength of the optical thickness here. From Figure I take that it is at $0.86 \mu \mathrm{m}$. Why? If the optical thickness is taken in the visible, the 550nm is a common wavelength to use. For the rest of the paper the authors use the $12.03 \mu \mathrm{m}$ optical thickness. I suggest using the $12.03 \mu \mathrm{m}$ here as well. Additionally, why is the $12.03 \mu \mathrm{m}$ wavelength chosen? It is one of the channels of course, but how strong does the optical thickness vary for the wavelength of the other channels?

16) Page 8, Line 26-28: Something about this paragraph is confusing and requires a better explanation. After reading it several times, I still cannot understand it in full. You point out that extreme values of the BT are smoothed out by the HRT effect. Therefore the difference between 1D and 3D BT should be smaller. As there is more scattering in channel $8.52 \mu \mathrm{m}$, one would expect smaller differences between $1 \mathrm{D}$ and $3 \mathrm{D}$ BT from the first conclusion. However, Figure 6 and your text show the opposite. This paragraph needs clarification. In addition, the choice of colors, the thickness of the lines and the scale of the $y$-axis makes this figure hard to read.

17) Page 9, Line 13: replace "smaller scattering" by "less scattering"

18) Page 9, Line 19: typo: quite instead of quitte 
19) Page 11, Line 21: delete "(FLIP average distance before absorption or before leaving the cloud)" - this is explained a few times already

20) Page 11, Line 22: typo: rapidly instead of rapidelly

Interactive

comment

21) Page 12, Line 4: typo: Nevertheless instead of Netherless

22) Page 12, Line 32: optical thickness at which wavelength?

23) Page 13, Line 12: "we chose to not show" - replace by "we chose not to show"

24) Page 14, Line 7: I fully understand that Monte Carlo simulations are very expensive in terms of computational time. However, as scattering is stronger in the $8.52 \mu \mathrm{m}$ channel and more horizontal transport of FLIPs between the column should occur, it might be worth adding this channel to the analysis? What result would be expected for the $8.52 \mu \mathrm{m}$ channel?

25) Page 14, Lines 20-22: Reformulate this sentence "In contrast, some lines of sight cross through small optical thicknesses..."

26) Page 14, Line 28: Remove "about"

27) Page 15, Line 5: typo: lige 
29) Conclusion: An outlook concerning the presented work would be beneficial for this section. The authors briefly state what will be shown in a Part 2 paper, however different wavelength channels are involved there.

As the choice of the cloud scene seems to have a larger impact on the off-nadir results, additional simulations (in future work) including different cirrus cloud fields might be one aspect. In addition, some discussion about the implications of the results for current cirrus cloud retrievals is wanting. How much would a satellite instrument with a resolution of $100-250 \mathrm{~m}$ improve current retrievals? One might discuss that in the context of earlier studies (e.g. Fauchez et al., 2015) where the BT differences of $10 \mathrm{~K}$ was related to ice crystal diameter and retrieved optical thickness. Is there a guess how much this improved resolution, with the following smaller differences in BT would improve the retrieval results? Currently, the conclusion section does not really show any conclusions. It only summarizes the presented work.

30) Figure 1, Caption: Delete "cirrus 1" and add "of the study"

31) Figure 2: Is the potential temperature and the equivalent potential temperature really the same?

32) Figure 3: Which optical thickness is shown in the figure?

33) Figure 4: Delete "cirrus 1"

34) Figure 5: Why do you use the optical thickness at $0.86 \mu \mathrm{m}$ here and $12.03 \mu \mathrm{m}$ in the 
following? The colors and especially the markers are hard to separate in this figure. One really has to zoom into the pdf.

35) Figure 6: The difference between the lines is hard to see, especially the red and pink colors are hard to differentiate. Also, the scale of the y-axis makes it difficult to see the differences properly. The authors might also consider plotting thicker lines.

36) Figure 7: The first sentence of the caption is challenging to understand.

37) Figure 8 and following: Please use a different color for the pink lines. Maybe green or orange?

38) Figure 9: delete the "t" after "to" at the end of he third line.

39) Figure 10: The lines in the upper row are hard to separate. I can see that you want to keep the values of the y-axis constant, but you might think of reducing it to 8 instead of 10 ? Maybe this would already help?

40) Table 1: remove "cirrus 1"

\section{Technical corrections}

Please see the "Specific Comments" section. 